\title{
FEASIBILITY STUDIES OF OPEN CHARM MEASUREMENTS WITH THE NA61/SHINE EXPERIMENT AT CERN-SPS
}

\author{
Yasir Ali ${ }^{\dagger}$, Pawee Staszel, Antoni Marcinek \\ Janusz BrzychczyK, Roman PŁaneta
}

The Marian Smoluchowski Institute of Physics, Jagiellonian University Reymonta 4, 30-059 Kraków, Poland

(Received September 26, 2013)

The results of feasibility studies for the $D^{0}$ meson (open charm) measurements by its decay into two daughter particles, pion and kaon, in central $\mathrm{Pb}-\mathrm{Pb}$ collisions at SPS energies are presented. To generate the physical input we use AMPT (A Multi-Phase Transport model) event generator. We employ GEANT4 application to describe particle transport through the experimental setup. The study is done assuming NA61/SHINE detector system supplemented with a future Vertex Detector (VD), which allows for precise track reconstruction at the target proximity. This precision is needed to select pion and kaon pairs that originate from the $D^{0}$ decays. The simulation results show that this measurement is feasible. This study also addresses the issue of vertex detector optimization with emphasis on the prospect of the development of a vertex detector based on CMOS technology.

DOI:10.5506/APhysPolB.44.2019

PACS numbers: $25.75 . \mathrm{Nq}, 29.40 . \mathrm{Wk}$

\section{Introduction}

It is widely recognized that the measurement of mesons containing heavy flavour is of high importance for better understanding of nucleus-nucleus reactions at relativistic energies. During the last four decades, an approach based on perturbative QCD (pQCD) was formulated and used for the interpretation of open and hidden charm experimental data [1]. The approach is based on the assumption that pQCD can describe charm production in elementary hadronic and nuclear collisions, and that charmonium creation

\footnotetext{
${ }^{\dagger}$ Corresponding author: yasir.ali@uj.edu.pl
} 
is due to the binding interaction between $c \bar{c}$ quarks. In the case of nucleonnucleus $(p+A)$ and nucleus-nucleus $(A+A)$ reactions, a created charmonium state encounters subsequent interactions with the surrounding matter [2]. For the open charm production, the standard approach assumes that a perturbatively produced charm quark forms a bound state with a lighter quark $(d, u$ or $s)$ [3]. It was pointed out that precise results on charmonium yield in $p+A$ are difficult to understand within the standard approach [4]. In 1997, Gorenstein and Gazdzicki [5, 6] observed that the $J / \psi$ yield in $A+A$ collisions is proportional to the pion multiplicity and that the data may be explained by statistical production of $J / \psi$ at the hadronization stage. A statistical approach was also developed for the open charm production. This model was based on the assumption that charm quark and anti-quark yields are generated in the partonic phase according to the available partonic phase space. The pQCD based and statistical estimates of open charm yields for central $\mathrm{Pb}+\mathrm{Pb}$ collisions at $158 \mathrm{AGeV}$ differ by a factor of about 30 [5]. The predicted system size dependence in these two approaches is also very different. Recently, it was pointed out by Satz [7] that only precise measurement of both open and hidden charm allows constructing observables that can provide information on the in-medium behaviour of quarkonia in a model independent way.

The charm-quark degrees of freedom are of vivid interest in the context of the phase transition between normal hadronic matter and the Quark-Gluon Plasma (QGP) [11, 12]. It was shown that $c \bar{c}$ meson states should no longer be formed in the coloured medium due to colour screening $[2,13]$. The $J / \psi$ mesons were measured at the top SPS energy by the NA38/NA50 and NA60 experiments. Figure 1 depicts the measured $J / \psi$ production as a function of centrality expressed as the number of participating nucleons, compared to pQCD predictions assuming normal nuclear absorption in the medium. The yield at the lower number of participants is consistent with pQCD, however, there is a significant drop at $N_{\text {part }} \approx 200$ [8]. This effect is known as the anomalous $J / \psi$ suppression. It was initially attributed to the onset of QGP formation in the high-density phase of nucleus-nucleus collision, however, other scenarios, such as interactions with comoving hadrons [14, 15], cannot be ruled out by this data. As suggested by Satz, the direct measurement of the open charm in nucleus-nucleus collisions would allow drawing final conclusions on the nature of described phenomenon in $J / \psi$ data.

The multiplicities of $\pi, \eta, \phi, K^{+}, K^{-}, D, \bar{D}$ and $J / \psi$ in the central $\mathrm{Au}+\mathrm{Au}$ collisions predicted by the Hadron String Dynamics (HSD) model are shown in Fig. 2 from AGS to RHIC energies. The predictions are based on the charm production cross sections fitted to the experimental $p+N$ and $\pi+N$ data assuming that the total charm production in $A+A$ reactions scales with the total number of nucleon-nucleon collisions [12]. It is seen 


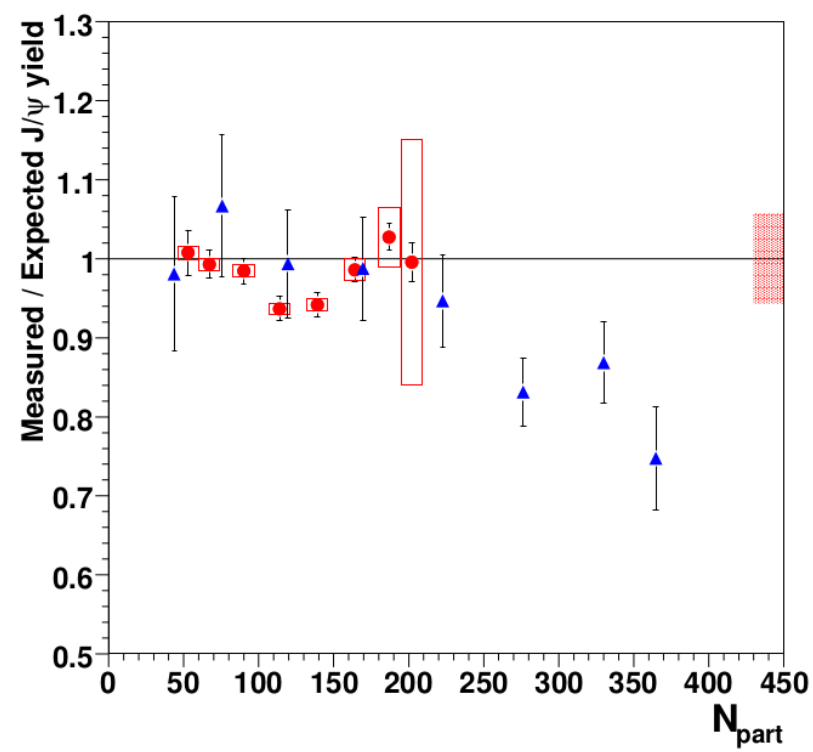

Fig. 1. $J / \psi$ suppression pattern measured in $\mathrm{In}+\mathrm{In}$ and $\mathrm{Pb}+\mathrm{Pb}$ collisions as a function of the number of participants $\mathrm{N}_{\text {part }}[8]$.

that the yields of the open charm are much higher than the yields of $J / \psi$, in particular, below the top SPS energy. This makes the experimental study of charm production in the open charm meson channel feasible not only for higher energies but also for lower energies, such as 30-40 A GeV.

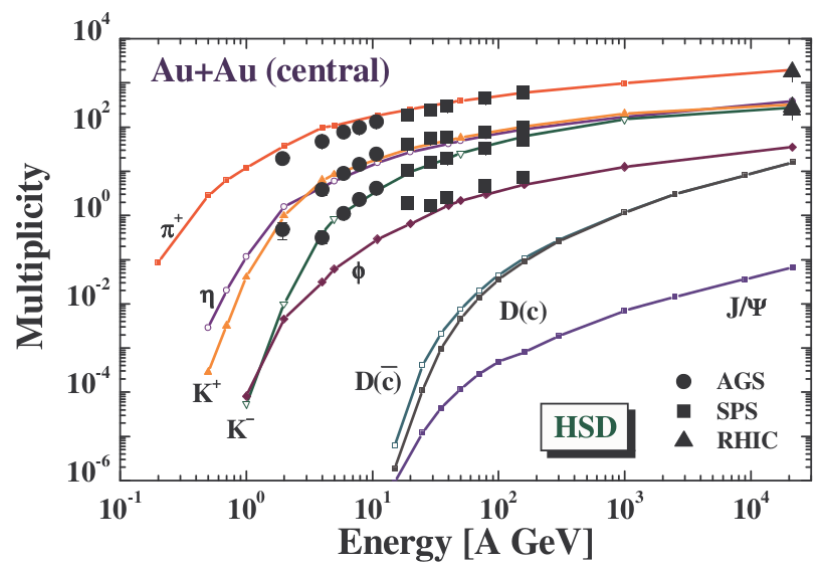

Fig. 2. Overview of the experimental meson multiplicities observed in central $\mathrm{Au}+\mathrm{Au}$ collisions as a function of the collision energy from SIS to RHIC energies [10]. The lines show the Hadron String Dynamics (HSD) model predictions. 


\section{NA61/SHINE experiment}

NA61/SHINE [16] is a fixed-target experiment at the CERN-SPS. The layout of the experimental setup is depicted in Fig. 3 [17]. It is a large acceptance hadron spectrometer, whose main components are inherited from the NA49 experiment [18].

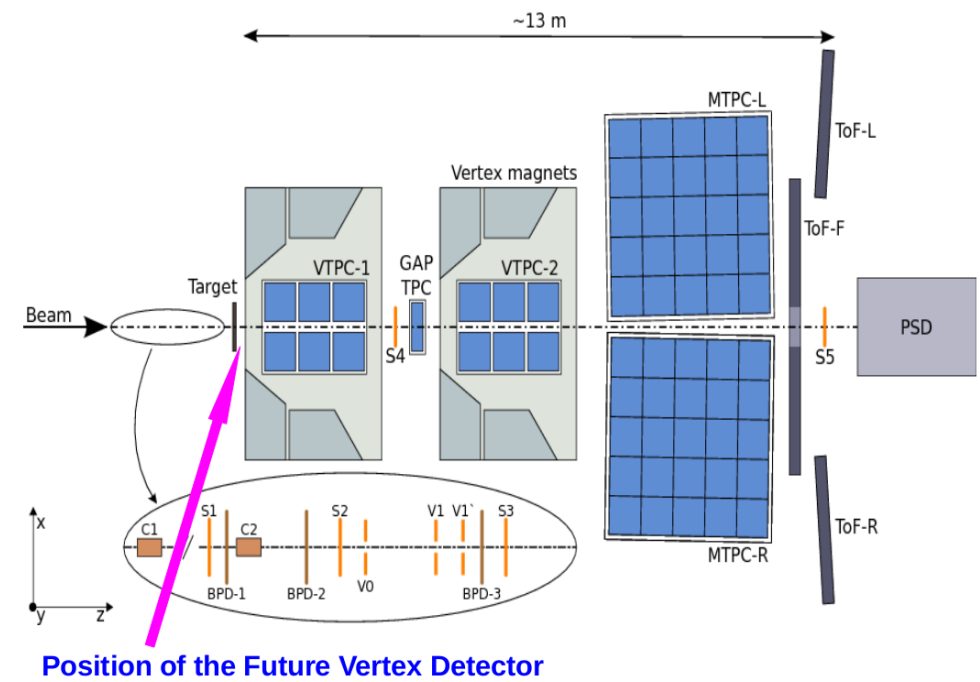

Fig. 3. The layout of the NA61/SHINE experimental set-up (top view, not to scale) with indicated position of the future vertex detector.

The setup includes the beam position detectors, Cherenkov counters and the scintillator stations located upstream of the target. These detectors provide information of timing, type and position of beam particles. For particle tracking, the experiment uses two Vertex Time Projection Chambers (VTPC1 and VTPC2), Gap TPC and two Main TPCs. The Time-of-Flight detectors, which are used for hadron identification, are located behind Main TPCs. The Projectile Spectator Detector (PSD) measures the energy of projectile spectators and delivers information on the collision centrality. The arrow drawn in the figure indicates the location of the future VD. It will be located downstream of the target, at the distance of about $50 \mathrm{~cm}$ to the front of the active VTPC1 volume.

\section{Feasibility studies of $D^{0}$ meson measurements}

There are several decay channels of open charm mesons listed in Table I [19]. Out of these, only the $D^{0} \rightarrow K^{-}+\pi^{+}$and $\overline{D^{0}} \rightarrow K^{+}+\pi^{-}$are twobody decays, which are easiest to measure in an experiment. This is why 
those channels are considered in this study. The measurement of the open charm at CERN-SPS energies is challenging due to low multiplicities and short life times of these particles. Of course, the other channels are not to be excluded from the future measurements, but due to their complexity they are left for later consideration.

TABLE I

Hadronic decay channels that are most promising for the reconstruction of open charm mesons [19].

\begin{tabular}{l|l|c|c}
\hline \hline Meson & \multicolumn{1}{|c|}{ Decay channel } & $c \tau$ & Branching ratio \\
\hline$D^{0}$ & $D^{0} \rightarrow K^{-}+\pi^{+}$ & $122.9 \mu \mathrm{m}$ & $(3.91 \pm 0.05) \%$ \\
$D^{0}$ & $D^{0} \rightarrow K^{-}+\pi^{+}+\pi^{+}+\pi^{-}$ & $122.9 \mu \mathrm{m}$ & $(8.14 \pm 0.20) \%$ \\
$D^{+}$ & $D^{+} \rightarrow K^{-}+\pi^{+}+\pi^{+}$ & $311.8 \mu \mathrm{m}$ & $(9.2 \pm 0.25) \%$ \\
$D_{s}^{+}$ & $D_{s}^{+} \rightarrow K^{+}+K^{-} \pi^{+}$ & $149.9 \mu \mathrm{m}$ & $(5.50 \pm 0.28) \%$ \\
$D^{*+}$ & $D^{*+} \rightarrow D^{0}+\pi^{+}$ & $\ldots$ & $(61.9 \pm 2.9) \%$
\end{tabular}

\subsection{Monte Carlo simulation}

We performed a feasibility study for the $D^{0}$ meson measurement by its decay into two daughter particles, $D^{0} \rightarrow K^{-}+\pi^{+}$, in central $\mathrm{Pb}-\mathrm{Pb}$ collisions at the top SPS energy of $158 A \mathrm{GeV}$ and the lower energy of $40 A \mathrm{GeV}$. As a physical input, we generated $200 \mathrm{k} \mathrm{0-10 \%} \mathrm{central} \mathrm{events} \mathrm{with} \mathrm{AMPT}$ (A Multi-Phase Transport model) event generator. It provides a good background description, which in the case of this study is a distribution of charged hadrons, mostly pions and kaons [20]. The AMPT model predicts the average multiplicity of 0.01 for $D^{0}+\bar{D}^{0}$ per central $\mathrm{Pb}+\mathrm{Pb}$ event. This value seems to be underpredicted with respect to PYTHIA results scaled to number of binary nucleon-nucleon collisions in central $\mathrm{Pb}+\mathrm{Pb}$ equal to 0.21 [21] as well as with respect to the prediction of the HSD model, which is 0.2 (see Fig. 2). Because HSD model was tuned to properly describe available $p+A$ and $\pi+\mathrm{A}$ charm production data at SPS energies [12], we scaled the AMPT multiplicities to the above prediction of the HSD model.

Within the given statistics, the AMPT event generator does not generate the open charm at the lower energy of $40 \mathrm{AGeV}$. However, we observe that the transverse mass slope parameter and the width of the rapidity distribution for kaons do not change by more than $10 \%$ going from $158 \mathrm{AGeV}$ to $40 A \mathrm{GeV}$. In order to describe the phase space for $D^{0}$ at the lower energy, we assumed changes in shapes of distributions similar to that observed for kaons. The total yield was taken as predicted by the HSD model. 
We employed GEANT4 to describe the particle transport through the NA61/SHINE experimental setup supplemented with the VD, which allows for precise tracking at the target proximity. The VD modelled in GEANT4 consists of four detection stations VDS1, VDS2, VDS3 and VDS4 located respectively at distances of $5 \mathrm{~cm}, 10 \mathrm{~cm}, 15 \mathrm{~cm}$, and $20 \mathrm{~cm}$ downstream of the target. The external transverse dimensions of the stations are $2 \times 4 \mathrm{~cm}^{2}$ (VDS1), $4 \times 8 \mathrm{~cm}^{2}$ (VDS2), $6 \times 12 \mathrm{~cm}^{2}$ (VDS3), $8 \times 16 \mathrm{~cm}^{2}$ (VDS4). These dimensions allow us to cover more than $99 \%$ of the acceptance of pions and kaons originating from the decay of $D^{0}$ mesons.

Each station has also a square hole $\left(3 \times 3 \mathrm{~mm}^{2}\right)$ in the central part, which allows the beam ions to go through without an interaction. The stations and the target are closed in the aluminium vessel as shown in Fig. 4. The vessel is equipped with thin front and back kapton windows, and filled with helium gas at atmospheric pressure to minimize multiple scattering of the produced particles. Each station consists of the layer of silicon $(50 \mu \mathrm{m})$, epoxy glue $(50 \mu \mathrm{m})$ and the carbon fiber $(300 \mu \mathrm{m})$. They represent the layer of MIMOSA-26 sensors with glue used to fix the sensors and the mechanical support itself. On the back side, we modelled thin water cooling tubes, so the mechanical support is integrated with the cooling system. This structure is based on the carbon fiber support developed at CERN for the upgrade of the ALICE vertex detector.

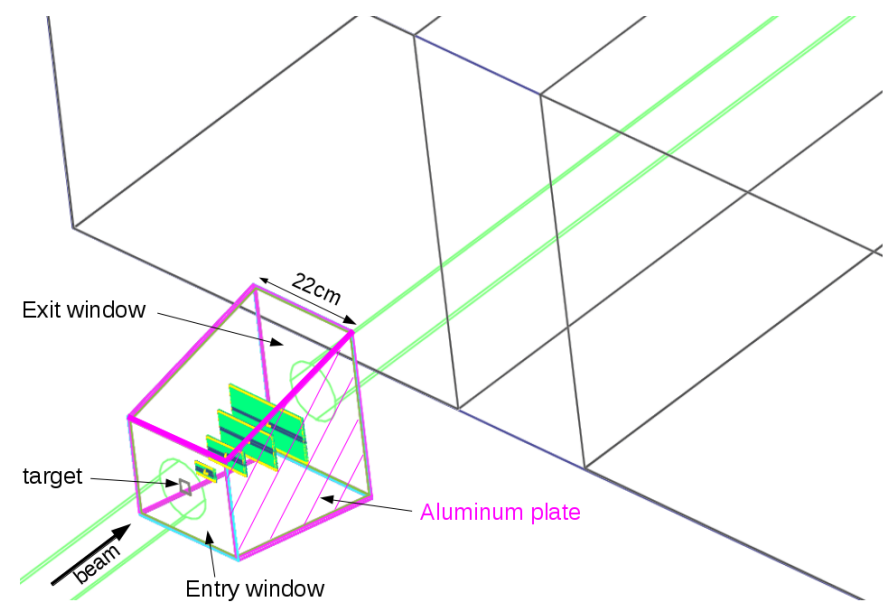

Fig. 4. The helium vessel containing four vertex detector stations, carbon fiber support and water cooling tubes.

The track reconstruction is based on the hits generated during particle transport through vertex detector stations, VTPC1 and VTPC2. Only the tracks accepted in VTPC detectors are considered in the subsequent analysis. The acceptance is defined in the similar way as it is done in the standard 
NA61/SHINE simulation, namely, we require that the track length in the active volume of VTPC1 and VTPC2 must be greater than $80 \mathrm{~cm}$. The momenta of the reconstructed tracks are smeared out according to the experimental momentum resolution [18]. The tracks reconstructed in VTPCs are extrapolated to the target region and the hits generated in VDS1-VDS4 are used to perform precise tracking. It is assumed that the position resolution for each station is $4 \mu \mathrm{m}$, which is consistent with the results obtained during beam tests of a telescope based on MIMOSA-26 sensors [22].

\subsection{Analysis and results}

The invariant mass distribution for kaon-pion pairs at the collision energy of $158 A \mathrm{GeV}$ is depicted in Fig. 5. As can be seen, the combinatorial background is few orders of magnitude higher than the $D^{0}+\overline{D^{0}}$ signal shown in grey/magenta colour.

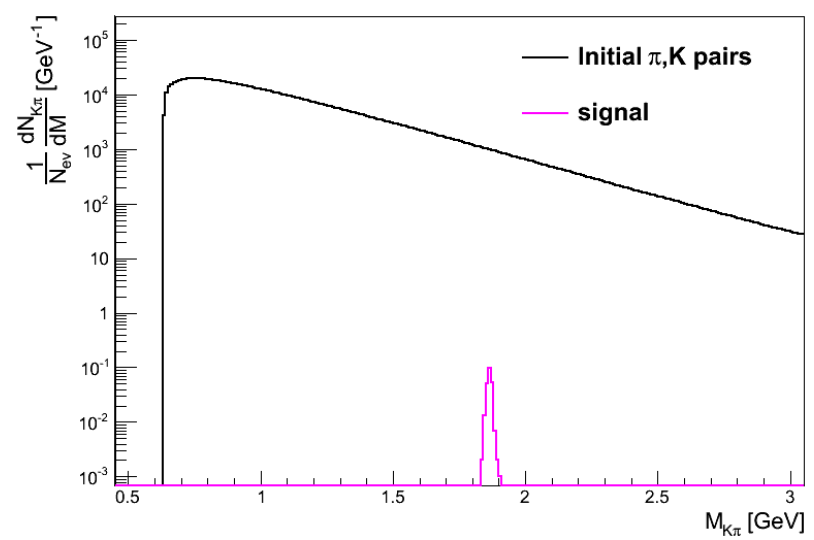

Fig. 5. The invariant mass distribution of pion-kaon pairs with the open charm signal at $1.86 \mathrm{GeV}$ in grey/magenta, at the collision energy of $158 \mathrm{AGeV}$.

$D^{0}$ mesons typically decay tens to few hundred microns downstream of their production point. To select pion and kaon pairs that originate from $D^{0}$ decays, one needs to reconstruct the decay vertex with a precision of about $10 \mu \mathrm{m}$. The TPC-based tracking system of the NA61/SHINE experiment, especially in the longitudinal direction, provides resolution several orders of magnitude worse. Therefore, a new, dedicated, high resolution vertex detector is required.

In order to suppress the large combinatorial background, four cuts were developed:

(i) cut on the track transverse momentum $p_{\mathrm{T}}$,

(ii) cut on the track impact parameter $d$, 
(iii) cut on the longitudinal position $V_{z}$ of the track pair vertex relative to primary interaction point,

(iv) cut on the parent particle impact parameter $D$.

The first two are single-particle cuts, while the two other are two-particle cuts. It should be stressed that cuts (ii)-(iv) require the precise tracking information at the target proximity from the proposed VD. Figures 6 and 7 show graphical definitions of the cut variables and their distributions respectively.
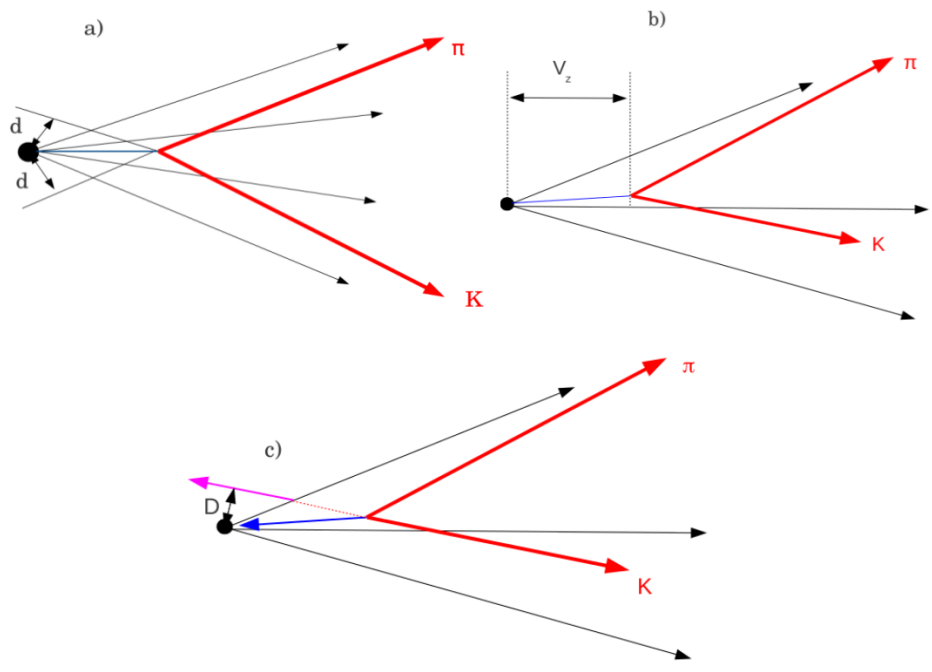

Fig. 6. Sketch of the $D^{0} \rightarrow K^{-}+\pi^{+}$decay topology with definitions of (a) track impact parameter $d,(\mathrm{~b})$ longitudinal position $V_{z}$ of the track pair vertex relative to primary interaction point and (c) impact parameter of the parent particle $D$. Thick gray/red tracks are the pion and kaon pairs while thin gray/black ones are particles produced in the primary interaction. The dot represents the primary vertex.

The top left diagram in Fig. 7 shows the transverse momentum distribution of signal and background tracks. The signal spectrum peaks at higher values of $p_{\mathrm{T}}$ than the background one, namely about $0.7 \mathrm{GeV} / c$ versus $0.2 \mathrm{GeV} / c$. This allows us to reject low $p_{\mathrm{T}}$ tracks as background-like ones.

Figure 6 (a) defines the track impact parameter $d$ as a distance of the closest approach between the track and the primary interaction vertex. Tracks originating from the primary interaction (thin gray/black arrows) contribute to the $d \approx 0$ peak with a spread related to multiple scattering in the target and detector material as well as to the limited detector resolution. Tracks originating from decays of $D^{0} \mathrm{~s}$ (thick gray/red arrows) are expected to have larger value of $d$, due to the displacement between primary interaction point 

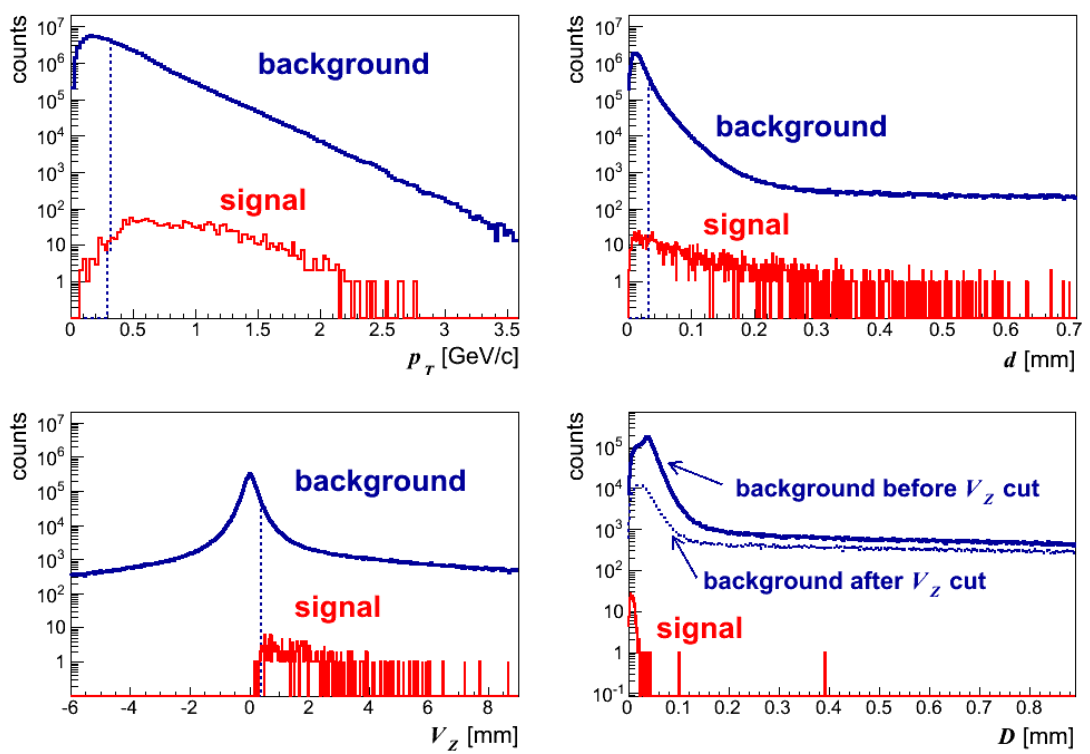

Fig. 7. Distributions of $p_{\mathrm{T}}, d, V_{z}$ and $D$ variables for background (thick/blue) and signal (thin/red). The reason for showing two background distributions for $D$ is explained in the text.

and $D^{0}$ decay vertex. Apart from tracks produced in the primary interaction, other secondary tracks (from other decays than $D^{0}$ ) contribute to the background. This yields the distributions of $d$ for background and signal tracks as seen in Fig. 7, top right plot. The contribution to the background from the primary interaction tracks as well as from products of fast decays, can be reduced by rejecting tracks with small $d$ values.

Figure 6 (b) defines $V_{z}$ as a longitudinal position of the track pair vertex relative to primary interaction point. As can be seen in the bottom left plot in Fig. 7, $V_{z}$ distribution for background pairs peaks strongly at zero, due to a huge contribution from pairs formed from primary interaction tracks. This contribution could be removed by rejecting low $V_{z}$ pairs.

Figure 6 (c) defines parent particle impact parameter $D$ as a distance of the closest approach between the primary interaction vertex and reconstructed trajectory of the non-measured directly, assumed particle (black/ blue arrow), which decays into a measured pair of tracks (thick gray/red arrows). This value is peaked at zero for both signal and background pairs see Fig. 7 , bottom right plot. For signal pairs, it is because the $D^{0}$ originates from the primary interaction vertex. For the background, the peak is correlated with the peak at $V_{z}=0$ and, as can be seen from the figure, applying the $V_{z}$ cut reduces the background at peak of $D$ by 2 orders of magnitude. 
The background has also a long tail, which is associated with pairs formed e.g. from a track that comes from the primary interaction point and another that originates from a decay. This tail can be removed by rejecting pairs with large $D$ values.

The cuts described above were optimized to obtain maximum value of signal to noise ratio (SNR). Their optimal values are shown as vertical lines in Fig. 7. In the analysis, only tracks that satisfy conditions $p_{\mathrm{T}}>0.4 \mathrm{GeV} / c$ and $d>40 \mu \mathrm{m}$ as well as pairs of tracks that satisfy $V_{z}>500 \mu \mathrm{m}$ and $D<22 \mu \mathrm{m}$ are accepted.

Figure 8 shows the initial invariant mass distribution (long dashed/black) at the collision energy of $158 \mathrm{AGeV}$ and the effect of subsequent application of the cuts, namely, after the cut on $p_{\mathrm{T}}$ (dashed/magenta), cut on $p_{\mathrm{T}}$ and $d$ (pale gray/yellow), cut on $p_{\mathrm{T}}, d$ and $V_{z}$ (dash-dotted/blue) and finally cut on $p_{\mathrm{T}}, d, V_{z}$ and $D$ (solid dark gray/red). When the complete set of cuts is applied, the $D^{0}+\overline{D^{0}}$ signal peak emerges at $D^{0}$ invariant mass of $1.86 \mathrm{GeV}$. With the developed background reduction strategy, the signal is suppressed by a factor of 1.8 , while the background in the signal region is reduced by a factor of $10^{6}$.

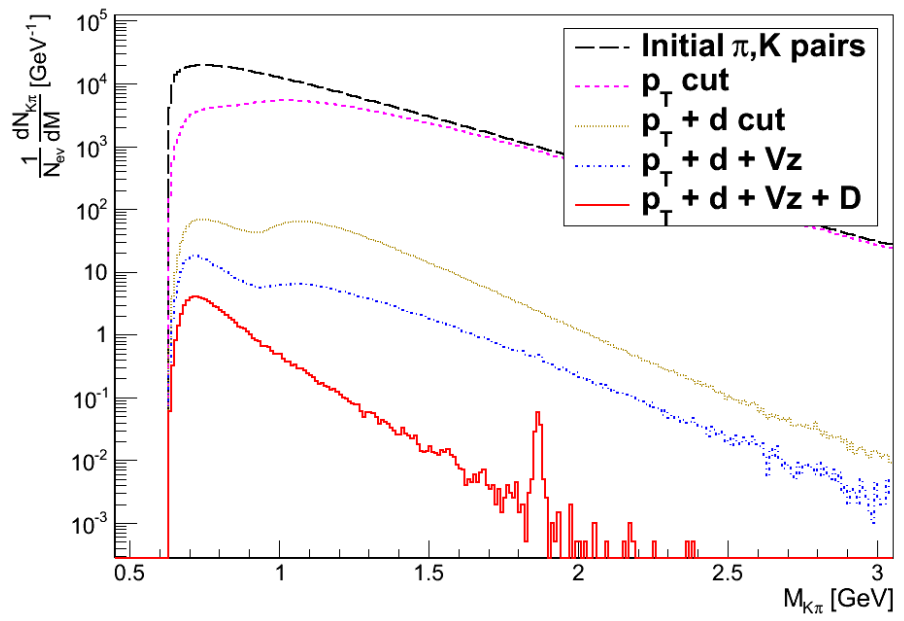

Fig. 8. The invariant mass distribution plot for pion and kaon pairs at the collision energy of $158 \mathrm{AGeV}$. Signal of open charm emerges at $1.86 \mathrm{GeV}$ with all the mentioned cuts applied.

So far, the analysis has assumed the perfect particle identification (PID), i.e. for each track the related specie type was known. The invariant mass distribution with all the cuts applied, assuming perfect PID, at the collision energy of $158 A \mathrm{GeV}$, is shown again in the left panel of Fig. 9. A fitted curve 

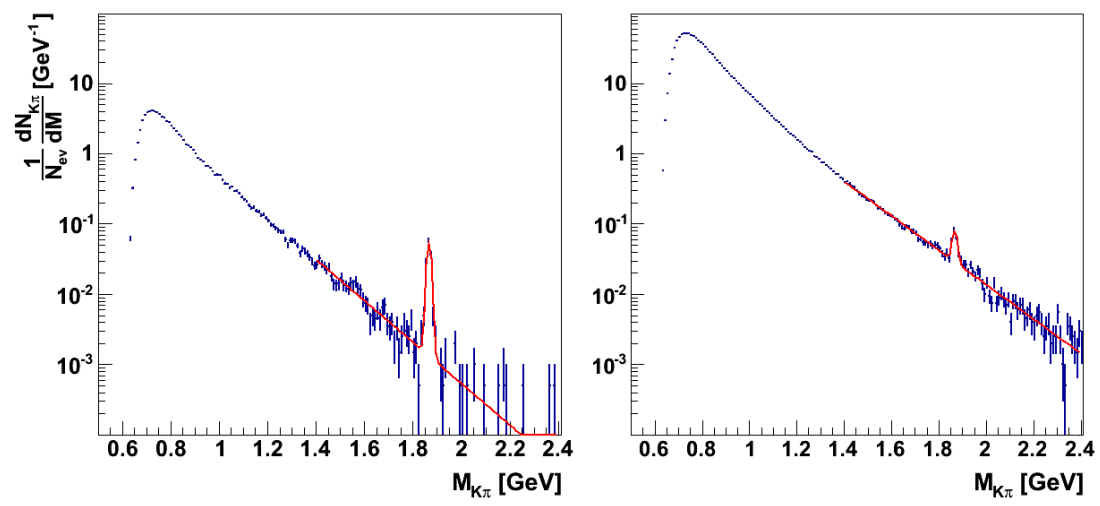

Fig. 9. The invariant mass of pion-kaon pairs after full background reduction. The curve represents fit with sum of exponential (background) and Gaussian functions ( $D^{0}$ signal). Left: analysis done using PID information, right: analysis that does not utilize the PID information (see the text for more explanation).

that represents a sum of exponential (background) and Gaussian (signal) functions are also shown. This curve is used to derive the $D^{0}+\overline{D^{0}}$ yields of the signal (S) and the background in the signal region (B). This plot is to be compared to the one in the right panel of Fig. 9, which presents results of an analysis that does not utilize the PID information. In this case for each unlike-sign pair of tracks, two hypotheses, corresponding to $D^{0}$ and $\overline{D^{0}}$, are tested - namely assuming that the negative track in the pair is kaon and the positive is pion and reversely. As can be seen from the comparison of the plots, the analysis without PID suffers from a much higher background. Nevertheless, it is feasible to extract the signal yield even in such an analysis. The reason to compare analyses with and without PID is that in the NA61/SHINE experiment, the exact identification on track by track basis is not possible in the whole phase space. Therefore, the real situation is going to be something between the two quoted analysis types. This means, that the measurement of the open charm in the NA61/SHINE experiment is feasible if the detector system is supplemented with a new vertex detector.

Table II shows expectations for open charm measurements at two energies available at CERN-SPS - $158 \mathrm{AGeV}$ discussed so far and the lower energy of $40 A \mathrm{GeV}$, for two analysis types - with and without PID. The signal and background yields obtained in the analysis were scaled up by a common factor to match the expectation from samples of $50 \mathrm{M}$ events, which is an anticipated event number collected during 1-2 months of NA61/SHINE data taking. As one can read from the table, the measurement of the open charm seems to be feasible even at the lower energy of $40 \mathrm{AGeV}$. 
Results of the simulation for the $D^{0}$ measurement in the decay channel $D^{0} \rightarrow$ $K^{-}+\pi^{+}$. The signal to noise ratio (SNR) and the total $D^{0}+\overline{D^{0}}$ yield $(\mathrm{S})$ are extrapolated to a sample of $50 \mathrm{M}$ central $\mathrm{Pb}+\mathrm{Pb}$ events.

\begin{tabular}{l|l|l|l|l}
\hline \hline Energy & $158 A \mathrm{GeV}$ & $158 A \mathrm{GeV}$ & $40 A \mathrm{GeV}$ & $40 A \mathrm{GeV}$ \\
\hline PID & perfect & no & perfect & no \\
S/B & 17 & 1.0 & 1.0 & 0.07 \\
SNR & 246 & 197 & 11.3 & 2.1 \\
S & $64 \mathrm{k}$ & $64 \mathrm{k}$ & $2 \mathrm{k}$ & $2 \mathrm{k}$
\end{tabular}

\section{Technology for the Vertex Detector}

The MIMOSA-26 sensor has been chosen as a basic detection element of the VD stations. The floor plan view of the MIMOSA-26 sensor is shown in Fig. 10. The chip is hosting a sensitive area of about $1.06 \times 2.12 \mathrm{~cm}^{2}$ with the pixel pitch equal to $18.4 \mu \mathrm{m}$, which gives $576 \times 1152=663.5 \mathrm{k}$ pixels per chip. Pixel columns are read out in parallel, row by row. The chip readout time is $115.2 \mu \mathrm{s}$. Each pixel includes an amplification and Correlated Double Sampling (CDS) units and each end of column is equipped with a discriminator. After the analogue to digital conversion, digital signals pass through zero suppression circuits. The digital signals are processed in

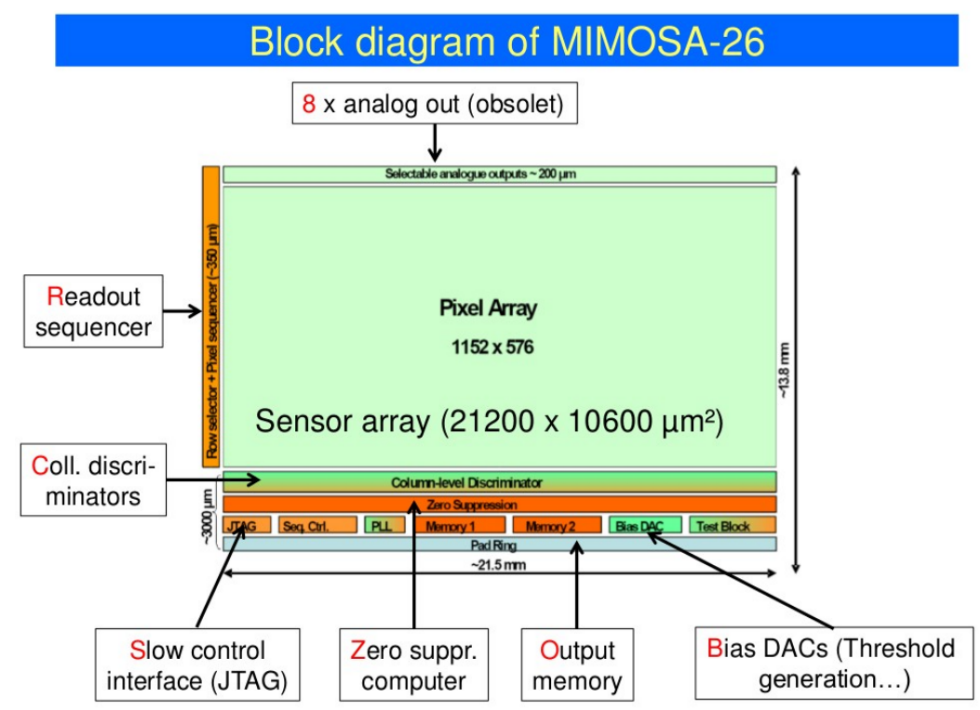

Fig. 10. Schematic view of the MIMOSA-26 displaying the basic architecture of the chip. 
parallel on 18 banks, then stored row by row in the memory. Two memory banks are implemented in the sensor to perform read and write operations simultaneously [23].

The selection of MIMOSA-26 was mostly driven by the very high hit occupancy in the stations found from simulations. The hit occupancy in the VDS1 generated by a single central $\mathrm{Pb}+\mathrm{Pb}$ collision at $158 \mathrm{AGeV}$ reaches values of about 5 hits $/ \mathrm{mm}^{2}$ /event in the innermost part of the detector. Such a high occupancy cannot be handled by existing micro-pattern gas detectors mostly because of their one-dimensional readout. For this technology, even for the hardly achievable strip pitch size at the level of $100 \mu \mathrm{m}$, the twoparticle separation for the indicated occupancy is not feasible. On the other hand, MIMOSA-26 offers high pixel granularity with a real 2-dimensional readout.

There are two sources of particles that will be collected during the sensor exposure time (which is $115.2 \mu \mathrm{s}$ ), namely particles produced in hadronic nucleus-nucleus interaction and $\delta$-electrons produced by beam ions passing through the target. To obtain more quantitative predictions on the two-particle separation, we generated sample of minimum bias $\mathrm{Pb}+\mathrm{Pb}$ collisions at $158 \mathrm{~A} \mathrm{GeV}$ to account for the first component, and to account for the component associated with $\delta$-electrons we simulated electromagnetic interactions of $\mathrm{Pb}$ beam ions passing through the $200 \mu \mathrm{m}$ thick $\mathrm{Pb}$ target. The anticipated beam intensity in the spill during data taking is $10^{5} \mathrm{~Hz}$, which leads to $500 \mathrm{~Hz}$ hadronic interactions in the target. From the numbers indicated above, one can get the probability of a single hadronic interaction collection in the single frame, which is about $5 \%$. At the same time, the sensor will collect on average $\approx 10$ bunches of $\delta$-electrons generated by passing beam ions. Even in a very challenging case, when one central and one peripheral $\mathrm{Pb}+\mathrm{Pb}$ events are collected in one frame, the estimated single hit occupancy at the most illuminated part of the sensor is $0.75 \%$. In the calculation we assumed that one particle hitting the sensor generates cluster containing on average 3 pixels [24]. This number shows that the random overlap of two clusters is very low $\left(<10^{-4}\right)$.

It is expected that during the data taking period, the VD will be exposed to high level of radiation. The sensor performance can be deteriorated by the non-ionizing energy loss (NIEL). Again, two sources of particles have to be considered, namely particles produced in hadronic nucleus-nucleus interaction and $\delta$-electrons. Based on the anticipated beam intensity and the interaction rates, the particle fluxes through the VD detector were calculated. Integrating the fluxes over 1 month (in 1-2 months NA61/SHINE can collect about $50 \mathrm{M}$ central $\mathrm{Pb}+\mathrm{Pb}$ events) and using the so-called displacement damage function [25], the fluencies of $2.6 \times 10^{10} n_{\mathrm{eq}} / \mathrm{cm}^{2}$ and $4.2 \times 10^{10} n_{\mathrm{eq}} / \mathrm{cm}^{2}$ in the most illuminated area of sensors were found for $\delta$-electrons and hadrons, respectively. The tests on proton beam of 
MIMOSA-26 sensors proved that these devices can handle fluencies up to $3 \times 10^{12} n_{\mathrm{eq}} / \mathrm{cm}^{2}$, which provides a large safety margin for using them in the NA61/SHINE application [26].

Another argument for selecting MIMOSA-26 chip was that these sensors can be thinned to $50 \mu \mathrm{m}$ thickness, which means that the sensor itself provides a small fraction of the total material budget as compared to the amount of material introduced by a mechanical support, cooling fluid and readout cabling. Table III summarizes the results obtained in this section and, for comprehensivity, shows limits of the performances related to alternative Hybrid and charge-coupled device (CCD) technologies. It can be seen that only MIMOSA-26 chips fulfils all the requirements imposed by the NA61/SHINE application.

TABLE III

The NA61/SHINE requirements and limits for different chip technologies. (1) and (2) refer to non-ionizing and ionizing doses respectively, per week of beam on target.

\begin{tabular}{l|l|l|l|l}
\hline \hline & \multicolumn{1}{c|}{ NA61 } & \multicolumn{1}{c|}{ Hybrid } & \multicolumn{1}{c}{ CCD } & \multicolumn{1}{c}{ MIMOSA-26 } \\
\hline Resolution & $<5 \mu \mathrm{m}$ & $30 \mu \mathrm{m}$ & $<5 \mu \mathrm{m}$ & $3.5 \mu \mathrm{m}$ \\
Mat. budg. & $\sim 0.1 \% \mathrm{X}_{0}$ & $\sim 1 \% \mathrm{X}_{0}$ & $\sim 0.1 \% \mathrm{X}_{0}$ & $0.05 \% \mathrm{X}_{0}$ \\
Rad. tol.(1) & $3 \times 10^{10} n_{\text {eq }} / \mathrm{cm}^{2}$ & $>10^{14} n_{\mathrm{eq}} / \mathrm{cm}^{2}$ & $<10^{9} n_{\mathrm{eq}} / \mathrm{cm}^{2}$ & $>10^{13} n_{\mathrm{eq}} / \mathrm{cm}^{2}$ \\
Rad. tol.(2) & $\sim 1 \mathrm{krad}$ & $>10 \mathrm{Mrad}$ & $\sim 1 \mathrm{Mrad}$ & $>300 \mathrm{krad}$ \\
Time res. & $\sim 100 \mu \mathrm{s}$ & $200 \mathrm{~ns}$ & $\sim 1000 \mu \mathrm{s}$ & $115.20 \mu \mathrm{s}$
\end{tabular}

\section{Summary}

Measurements of mesons containing heavy flavour are important for better understanding of nucleus-nucleus reactions at relativistic energies. In this respect, there are experimental initiatives which measure charmonia states at SPS energies but so far there have been no direct open charm measurements at SPS energies. Thus, results of the feasibility study for the $D^{0}$ meson (open charm) measurements by its decay into two daughter particles, pion and kaon, in central $\mathrm{Pb}-\mathrm{Pb}$ collisions at the top SPS energy of $158 A \mathrm{GeV}$ and lower energy of $40 \mathrm{AGeV}$, were presented. AMPT model was used to generate physical input and GEANT4 was employed to describe particle transport through the experimental setup. Monte Carlo simulation results show that this measurement is feasible with the NA61/SHINE experiment, if it is supplemented with a new, high precision, Vertex Detector (VD), which allows the precise track reconstruction at the target proximity. This VD will be based on MIMOSA-26 sensors which fulfil all the requirements imposed by the NA61/SHINE application. 
This contribution reports on the technical work motivated by the future experimental programme of the NA61/SHINE Collaboration at CERN-SPS. Authors gratefully acknowledge the contribution of NA61/SHINE to the results presented. This work is supported by the Foundation for Polish Science - MPD program, co-financed by the European Union within the European Regional Development Fund and the National Science Centre of Poland, grant UMO-2012/04/M/ST2/00816.

\section{REFERENCES}

[1] G. Sterman et al., Rev. Mod. Phys. 67, 157 (1995).

[2] T. Matsui, H. Satz, Phys. Lett. B178, 416 (1986).

[3] R. Vogt, 1999, http://hips.web.cern.ch/HIPS/charm/dec99/vogt/

[4] J. Aichelin, 1999, http://hips . web.cern.ch/HIPS/charm/dec99/aichelin/

[5] M. Gaździcki, M.I. Gorenstein, Acta Phys. Pol. B 30, 2705 (1999).

[6] M.I. Gorenstein, Phys. Rev. C60, 054903 (1999).

[7] H. Satz, arXiv:1303.3493v2 [hep-ph].

[8] E. Scomparin [NA60 Collab.], Recent results from the NA60 experiment, 2009.

[9] M. Masera [NA50 Collab.], J. Phys. G: Part. Phys. 27, 677 (2001).

[10] O. Linyk et al., Int. J. Mod. Phys. E17, 1367 (2008) [arXiv:0808:1504 [nucl-th]].

[11] U. Heinz, Nucl. Phys. A661, 140c (1999).

[12] W. Cassing, E.L. Bratkovskaya, A. Sibirtsev, Nucl. Phys. A691, 753 (2001).

[13] H. Satz, Prog. Rep. 310, 197 (1999).

[14] W. Cassing, E.L. Bratkovskaya, Phys. Rep. 308, 65 (1999).

[15] A. Capella, E.G. Ferreiro, A.B. Kaidalov, Phys. Rev. Lett. 85, 2018 (2000).

[16] The NA61/SHINE homepage, http://na61.web.cern.ch

[17] T. Czopowicz [NA61/SHINE Collab.], Status and Plans of the NA61/SHINE Physics Program, ISMD Conference, 2011.

[18] S. Afanasiev et al., Nucl. Instrum. Methods Phys. Res. A430, 210 (1999).

[19] C. Amsler et al. [Particle Data Group], Phys. Lett. B667, 1 (2008) and 2009 partial update for 2010 edition.

[20] Zi-Wei Lin et al., Phys. Rev. C72, 064901 (2005).

[21] P. Braun-Munzinger, J. Stachel, Phys. Lett. B490, 196 (2000).

[22] J. Baudit et al., "First test results of MIMOSA-26, a fast CMOS sensor with integrated zero suppression and digitized output", IEEE Nuclear Science Symposium, Orlando, USA, October 2009. 
[23] MIMOSA-26 User Manual, Institut Pluridisciplinaire Hubert Curien IN2P3-CNRS.

[24] L. Qiyan, Poster Presentation, 20th International Conference CHEP, 2013.

[25] A. Vasilescu, ROSE Internal Note, ROSE/TN/97-2, 1997.

[26] M. Winter, CBM Progress Report, GSI/FAIR, 2010. 